\title{
Assessment of noninvasive, percutaneous hemoglobin measurement in pregnant and early postpartum women
}

This article was published in the following Dove Press journal:

Medical Devices: Evidence and Research

15 January 2014

Number of times this article has been viewed

\author{
Atsushi Yoshida' \\ Keiko Saito ${ }^{2}$ \\ Kenji Ishii ${ }^{2}$ \\ Isao Azuma ${ }^{2}$ \\ Hidenori Sasa' \\ Kenichi Furuya' \\ 'Department of Obstetrics and \\ Gynecology, National Defense \\ Medical College, Tokorozawa, Japan; \\ ${ }^{2}$ Department of Obstetrics and \\ Gynecology, Nishisaitama-Chuo \\ National Hospital, Tokorozawa, Japan
}

Correspondence: Atsushi Yoshida Department of Obstetrics and Gynecology, National Defense Medical College, 3-2 Namiki, Tokorozawa, Saitama 359-85 I3, Japan

Tel +8I 42995 I687

Fax +81429965213

Email ghh03464@nifty.ne.jp
Purpose: Using the Pronto- $7^{\circledR}$ analyzer, we measured percutaneous hemoglobin ( $\mathrm{SpHb}$ ) noninvasively in pregnant and early postpartum women, and assessed the accuracy of the measurements by comparing them with laboratory measurements of hemoglobin.

Methods: We obtained SpHb measurements from 193 pregnant women, 269 early postpartum women, and 76 nonpregnant women. A laboratory total hemoglobin (tHb) measurement, from venous blood sampling, was obtained immediately prior to the $\mathrm{SpHb}$ measurement. The total number of measurements obtained from the nonpregnant, pregnant, and postpartum women was 76, 438, and 347, respectively.

Results: The mean biases ( $\mathrm{SpHb}-\mathrm{tHb})$ among the nonpregnant, first trimester, second trimester, third trimester, and early postpartum women were $-0.20,0.19,1.01,1.32$, and $1.10 \mathrm{~g} / \mathrm{dL}$, respectively. The Bland-Altman comparison showed neither the tendency of a fixed bias nor proportional biases among the measurements in the category of nonpregnant and first trimester women. But in the second and third trimester and postpartum category, a significant fixed bias was noted, without any tendencies of proportional bias.

Conclusion: In this study, we found higher hemoglobin values with the Pronto-7 analyzer than were measured in the laboratory. We consider that the device has certain limitations in obstetrical utility and requires further modifications for use in the perinatal period.

Keywords: anemia, pregnancy, hemoglobin, noninvasive

\section{Introduction}

During pregnancy, dilutional anemia is often seen. This anemia is due to the relatively large increase in the extracellular fluid volume in comparison with the increase in the red blood cell production that occurs as a normal effect of pregnancy. However, severe anemia should be treated. Therefore, it is necessary to measure hemoglobin levels and check the severity of anemia during pregnancy. Although the number of maternal death due to postpartum hemorrhage has been decreasing in the USA ${ }^{1}$ and $\mathrm{UK},{ }^{2}$ severe hemorrhage still remains one of the highest causes of maternal morbidity. ${ }^{3}$ Also, in Japan, postpartum hemorrhage is the most common cause of maternal death, ${ }^{4}$ and the percentage of maternal deaths caused by postpartum hemorrhage has even increased, from $4.7 \%$ to $24.6 \%$ between 1995 and $2003 .{ }^{5}$ More recently, it was reported that $23 \%$ of the 83 maternal deaths from 2010 to 2012 were caused by a massive obstetric hemorrhage. ${ }^{6} \mathrm{~A}$ loss of blood at delivery is inevitable, and the accurate estimation of blood loss is difficult because blood is often mixed with amniotic fluid and sometimes, with urine and surgical sponges. Accordingly, the accurate evaluation of the severity of maternal postpartum anemia by estimated blood 
loss is very difficult. The clinical estimation derived from the vital signs and visual estimation of the amount of blood loss is a simple method, but this method has been reported to have a tendency toward underestimation. ${ }^{7}$ It would be very beneficial if the hemoglobin concentration could be measured in "real time" and noninvasively, during pregnancy or postpartum period.

Recently, a noninvasive device for percutaneous hemoglobin $(\mathrm{SpHb})$ measurement has been developed that relies on infrared absorption. The usefulness of $\mathrm{SpHb}$ measurement has been reported in various diseases or clinical conditions. ${ }^{8-10}$ The Pronto- $7^{\circledR}$ (Masimo Corporation, Irvine, CA, USA) emits infrared beams of seven different wavelengths simultaneously and it measures each hemoglobin with different absorption characteristics. Then, using spectrophotometric analysis, the Pronto-7 analyzer allows an on-the-spot measurement of $\mathrm{SpHb}$. The Pronto-7 is a small device with good portability, and the measurement time is as short as about 40 seconds. The performance data published by the Masimo Corporation are as follows: ${ }^{11}$

- The mean bias $(\mathrm{SpHb}-\mathrm{tHb})$ is $-0.02 \mathrm{~g} / \mathrm{dL}$

- One standard deviation of the bias is $0.99 \mathrm{~g} / \mathrm{dL}$

- For measurements between 6-18 g/dL, greater than $95 \%$ of $\mathrm{SpHb}$ readings are within $2 \mathrm{~g} / \mathrm{dL}$ of the reference laboratory total hemoglobin ( $\mathrm{tHb}$ ) value.

These performance data do not include measurement data on pregnant and postpartum subjects. Few reports have mentioned $\mathrm{SpHb}$ measurements in pregnant or postpartum women. To our knowledge, no major report has discussed the accuracy of $\mathrm{SpHb}$ measurements during pregnancy or the postpartum period.

In this study, we measured $\mathrm{SpHb}$ in pregnant and early postpartum women, using the Pronto- 7 and assessed the accuracy of the measurements by comparing them with laboratory hemoglobin measurements.

\section{Materials and methods}

The study was designed as a prospective cohort study, aiming to assess the accuracy of the Pronto-7 analyzer in obstetrical use. This study was conducted in 2012, at the Nishisaitama-Chuo National Hospital (Tokorozawa, Saitama, Japan) and was approved by the research ethics committee of the hospital.

Pregnant and postpartum women were asked to participate in this study, at the time of their routine blood tests. Nonpregnant subjects were requested to enroll in the study when they presented for blood testing for other medical indications. Nonpregnant medical staff members of the hospital also took part in the study, at the time of their regular health checkup. Women with significant deformities or degenerative changes of fingers were excluded. The $\mathrm{SpHb}$ measurement was obtained from 193 pregnant (6-40 weeks) Japanese women and 269 early (day 0-7) postpartum Japanese women. All the subjects gave informed consent prior to the measurements. A total of 438 measurements were obtained from 193 pregnant women, and 347 measurements were obtained from 269 postpartum women. In 58 women, measurements were made both in the pregnant and postpartum periods. In 76 nonpregnant women, 90 measurements were made as controls. The $\mathrm{SpHb}$ values were recorded using the Pronto-7. The $\mathrm{SpHb}$ probe was attached to the index, middle, or ring finger of either hand of the subjects. An approved black cloth shield was used to cover the sensor to avoid optical interference. The $\mathrm{SpHb}$ measurement was made in the supine or sitting position. The Pronto- 7 also provides a measurement of the percutaneous oxygen saturation $\left(\mathrm{SpO}_{2}\right)$ value, pulse rate $(\mathrm{PR})$, and perfusion index (PI) value. These values were also recorded at the time of the $\mathrm{SpHb}$ measurement.

Venous blood sampling for the measurement of laboratory $\mathrm{tHb}$ was done immediately before the $\mathrm{SpHb}$ measurement. The measurement of $\mathrm{tHb}$ was performed in the Nishisaitama-Chuo National Hospital Central Laboratory, using either of the following equipment: the XE-2100TM Hematology System (Sysmex Corporation, Kobe, Japan) or the XS-1000i'TM Automated Hematology Analyzer (Sysmex Corporation). A good correlation between the values of the Sysmex XS-1000i and the values of the XE-2100 has already been verified. ${ }^{12}$

\section{Statistical analysis}

The patient characteristics in each group (first, second, and third trimesters of pregnancy, postpartum, and nonpregnant) were summarized with descriptive statistics. The results were expressed as the mean \pm standard deviation (SD) (range), or as number (\%), as appropriate. In each group, the bias, calculated as the difference between the two measurements $(\mathrm{SpHb}-\mathrm{tHb})$, was obtained. In each group, the distribution of the bias was well fit to a normal distribution, but the variance of each group was not considered to be equal. Therefore, we used Scheffe's $F$-test for multiple comparisons of the parametric data. We assessed the relationship between the bias and the gestational age or postpartum period. Simple regression analysis was used for the correlative analysis. Using the Bland-Altman method, we made a difference plot for 
the analysis of the agreement between the two different hemoglobin measurement methods.

The statistical analysis was performed with Microsoft ${ }^{\circledR}$ Excel 2010 Japanese Version (Microsoft Japan, Tokyo, Japan) and Statcel version 3 (OMS Publishing Inc, Saitama, Japan). In the analysis described above, a $P$-value less than 0.01 was considered statistically significant.

\section{Results}

All the enrolled subjects completed the study. The characteristics of the subjects in each group were shown in Table 1.

Figure 1 shows the scattergram of all the measurements ( $\mathrm{n}=875)$. A significant positive correlation $(r=0.74, P<0.01)$ was noted between the $\mathrm{SpHb}$ measured by the Masimo Pronto-7 and the venous laboratory $\mathrm{tHb}$. The majority of the plots were located under the hatched line $(\mathrm{SpHb}=\mathrm{tHb})$, indicating the tendency of $\mathrm{SpHb}>\mathrm{tHb}$. However, each group had dissimilar distributions, suggesting that the bias had to be compared by group.

The bias in each group was shown in Figure 2. The bias in the nonpregnant group was $-0.20 \pm 1.20 \mathrm{~g} / \mathrm{dL}$. The biases in the first, second, and third trimesters of pregnancy and the postpartum period were $0.19 \pm 1.01,1.01 \pm 1.22,1.32 \pm 1.07$, and $1.10 \pm 1.13 \mathrm{~g} / \mathrm{dL}$, respectively. By using Scheffe's $F$-test, a significant difference was noted between the groups shown in Figure 2. As shown in Figure 2, the results of the biases can be divided into two major categories: (A) those who were nonpregnant and in the first trimester of pregnancy $(n=200)$; and (B) those in the second and third trimester of pregnancy and postpartum period $(n=675)$. Figure 3 shows scattergrams of the $\mathrm{SpHb}$ and laboratory $\mathrm{tHb}$ of the two categories. In the former category, the plots were located around the broken line
$(\mathrm{SpHb}=\mathrm{tHb})($ Figure 3A). In the latter category, the majority of the plots were located under the line $(\mathrm{SpHb}=\mathrm{tHb})$, indicating the tendency of $\mathrm{SpHb}>\mathrm{tHb}$ (Figure 3B).

The Bland-Altman comparison is thought to be a good method for comparing the accuracy of a new measurement method with the conventional "gold standard" method. ${ }^{13}$ Figure 4 demonstrates the Bland and Altman comparison of the $\mathrm{SpHb}$ and $\mathrm{tHb}$ of the two categories. The horizontal axis shows the average of the $\mathrm{SpHb}$ and $\mathrm{tHb}([\mathrm{SpHb}+\mathrm{tHb}] / 2)$, and the vertical axis shows the bias. The horizontal lines are drawn at the mean bias. The magnitudes of the biases are shown as the location of the horizontal line (fixed bias). If the magnitude of the bias depends on the measure of hemoglobin, a tendency will be shown in the Bland-Altman scattergram (proportional bias). In the category $\mathrm{A}$, the mean bias was -0.07 , and the $95 \%$ confidence interval contained zero $(-0.15$ to 0.01$)$. The plots were scattered almost everywhere, and the regression coefficient of the regression line (chained line) was $0.194(P>0.05)$. Therefore, we can see no tendency of the fixed bias or proportional bias in category A. On the other hand, in category B, the mean bias was 1.16 , and the 95\% confidence interval did not contain zero (1.08-1.24); the plots were scattered almost everywhere, and the regression coefficient of the regression line (chained line) was $0.087(P>0.05)$. We can see a significant fixed bias but no tendency of proportional bias in category $\mathrm{B}$.

\section{Discussion}

The laboratory measurement of hemoglobin requires transport of the samples, thus delaying the process. The potential improvement in patient care provided with a noninvasive method for measuring hemoglobin could be important as it

Table I Characteristics of the subjects in each group

\begin{tabular}{|c|c|c|c|c|c|}
\hline & \multirow[t]{2}{*}{ Nonpregnant } & \multicolumn{3}{|l|}{ Pregnant } & \multirow[t]{2}{*}{ Postpartum } \\
\hline & & First trimester & Second trimester & Third trimester & \\
\hline Number of subjects & 76 & & 193 & & $269 * *$ \\
\hline Number of measurements & 90 & 110 & 110 & 218 & 347 \\
\hline Age $(y r)^{*}$ & $4 I \pm 12(14-74)$ & $3 I \pm 6(|8-4|)$ & $32 \pm 5(19-42)$ & $32 \pm 6(14-74)$ & $32 \pm 5(17-44)$ \\
\hline Nulliparity (\%) & I7.I & 45.5 & 46.4 & 32.6 & $33.1 * * *$ \\
\hline Gestational age (weeks)* & - & $10 \pm 2(6-14)$ & $26 \pm 2(15-28)$ & $35 \pm 2(29-40)$ & $3 \pm 2(0-8) * * * *$ \\
\hline Height $(\mathrm{cm})^{*}$ & $158 \pm 6(142-173)$ & $158 \pm 6(14 \mid-173)$ & $159 \pm 5(145-172)$ & $158 \pm 5(\mid 42-172)$ & $158 \pm 5(142-173)$ \\
\hline Weight $(\mathrm{kg})^{*}$ & $58 \pm I I(40-95)$ & $55 \pm 9(42-86)$ & $55 \pm I 1(45-107)$ & $54 \pm 9(36-105)$ & $52 \pm 8(42-105)$ \\
\hline $\mathrm{SpHb}(\mathrm{mg} / \mathrm{dL})^{*}$ & 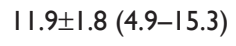 & $12.6 \pm 1.0(\mid 0.7-14.5)$ & $12.0 \pm I .3(8 .|-| 5.7)$ & $12.2 \pm 1.3(8.8-17.6)$ & 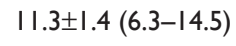 \\
\hline $\mathrm{tHb}(\mathrm{mg} / \mathrm{dL})^{*}$ & $12 . I \pm 2.1(6.2-\mid 5.2)$ & $|2.4 \pm I|.(10.0-15.2)$ & $11.0 \pm 1.2(6.9-13.5)$ & $10.8 \pm 1.1(8.2-14.7)$ & $10.2 \pm 1.4(4.6-13.8)$ \\
\hline $\mathrm{PI}(\%)^{*}$ & $5.8 \pm 3.7(0.8-20)$ & $7.8 \pm 4.8(0.93-20)$ & $8.5 \pm 4.3(0.8|-| 8.6)$ & $10.4 \pm 4.9(0.68-20)$ & $10.7 \pm 4.9(0.36-20)$ \\
\hline PR (beats per minute)* & $77 \pm 10(54-101)$ & $78 \pm 12(60-107)$ & $84 \pm 13(57-117)$ & $86 \pm 15(54-125)$ & $75 \pm 13(50-125)$ \\
\hline $\mathrm{SpO}_{2}(\%)^{*}$ & $98.3 \pm 1.2(94-100)$ & $98.4 \pm 1.0(96-100)$ & $98.1 \pm 1.02(95-100)$ & $97.7 \pm 1.0(93-100)$ & $97.4 \pm 1.4(874-100)$ \\
\hline
\end{tabular}

Notes: *Values are expressed as mean \pm SD (range); **fifty-eight postpartum women were measured also during pregnancy; ***primiparity; ****postpartum days.

Abbreviations: $\mathrm{PI}$, perfusion index; PR, pulse rate; SD, standard deviation; SpHb, percutaneous hemoglobin; $\mathrm{SpO}_{2}$; partial pressure of oxygen in arterial blood; tHb, total hemoglobin. 


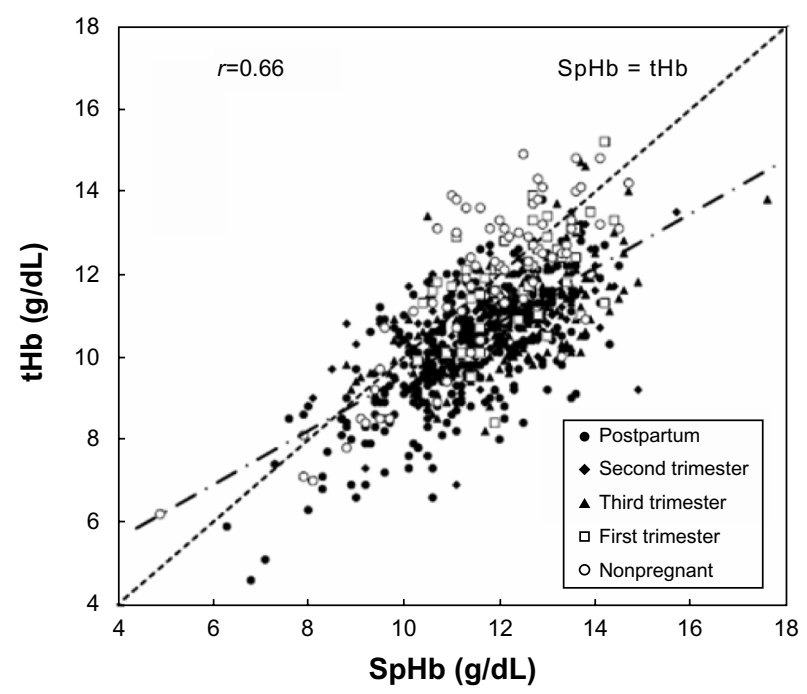

Figure I Scattergram of laboratory $\mathrm{tHb}$ and $\mathrm{SpHb}$ measurements. Note: The majority of the plots were located under the hatched line $(\mathrm{SpHb}=\mathrm{tH} b)$. Abbreviations: $\mathrm{SpH}$, percutaneous hemoglobin; tHb, total hemoglobin.

gives the result more rapidly, decreases exposure to potential biohazards, and finally, reduces pain and discomfort to the patient.

In this prospective study, we observed variable biases and a wide $\mathrm{SD}$ (or limits of agreement) between the $\mathrm{SpHb}$ and $\mathrm{tHb}$ measurement in the second and third trimesters of pregnancy and the early postpartum period. As shown in Figure 4B, the fixed bias in category B was noted, with no tendency of proportional bias. This result suggests that the variation of the biases is independent of the hemoglobin level.

Previously, a few reports mentioned the accuracy of $\mathrm{SpHb}$ during pregnancy or postpartum period. Butwick et al

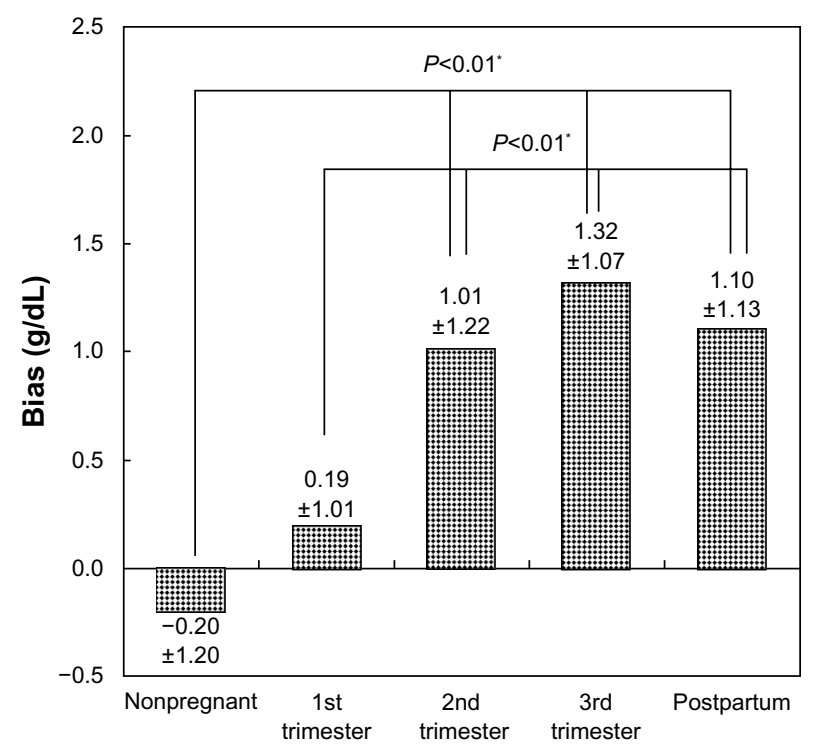

Figure 2 The bias in each group. Note: $*$ Significant difference $(P<0.0 \mathrm{I})$. reported the bias between the $\mathrm{SpHb}$ and $\mathrm{tHb}$, in patients undergoing elective cesarean section $(\mathrm{C} / \mathrm{S}) .{ }^{14}$ They compared the $\mathrm{SpHb}$ and laboratory tHb in 50 patients at baseline (before the fluid preloading in the preoperative period), during the immediate postoperative period (within 10 minutes of arrival in the recovery unit), and 24 hours after completion of the $\mathrm{C} / \mathrm{S}$, using the Masimo rainbow $\mathrm{SET}^{\circledR}$ Radical-7 (Masimo Corporation) pulse CO-Oximeter. In their report, significant positive biases at baseline (bias $=1.22$ ) and at 24 hours post-C/S (bias $=1.36$ ) were noted, but the bias immediately post-C/S was smaller (bias $=0.14$ ). In the current study, we used the Masimo Pronto-7, which adopted a similar technology to the Radical-7. The mean biases at baseline and at 24 hours post-C/S in their study were close to our values for the second or third trimester of pregnancy and the early postpartum period. The researchers did not clearly explain the reason why the bias in the immediate post$\mathrm{C} / \mathrm{S}$ period was smaller than those at baseline and 24 hours post-C/S. They speculated that the bias in the immediate post-C/S period may have been influenced by perioperative changes in the cardiac indices and intravascular volume. In general, the most dynamic change of cardiovascular function in the perinatal period is thought to take place at the moment of delivery. The researchers measured the $\mathrm{SpHb}$ and laboratory $\mathrm{tHb}$ for the immediate postoperative period within 10 minutes of arrival in the recovery unit, but they did not describe the precise time of the measurement with respect to the delivery. In our hospital, it usually takes almost 1 hour from $\mathrm{C} / \mathrm{S}$ delivery to the arrival in the recovery room. In our current study, we have only two data within 1 hour from delivery, but the biases of the two cases were 1.8 and 0.7 .

Butwick et al compared their results with those of previous studies in nonobstetric surgeries, ${ }^{15,16}$ and they described that the values for the biases at baseline and 24 hours post-C/S were higher than those in the previous studies of nonpregnant patients. They speculated that the gestational status may have influenced the accuracy of the $\mathrm{SpHb}$. However, they did not clearly describe the direct mechanism for how pregnancy influences $\mathrm{SpHb}$ measurement. They concluded that the device they used in their study had limitations in clinical utility and required modifications for obstetrical use.

In the current study, increased biases were noted, not only in the perioperative period but also, in the second and third trimesters of pregnancy and the early postpartum period. We speculate that $\mathrm{SpHb}$ measurement results may be affected by the circulatory status during pregnancy because the elevation of the bias in the first trimester was 
A

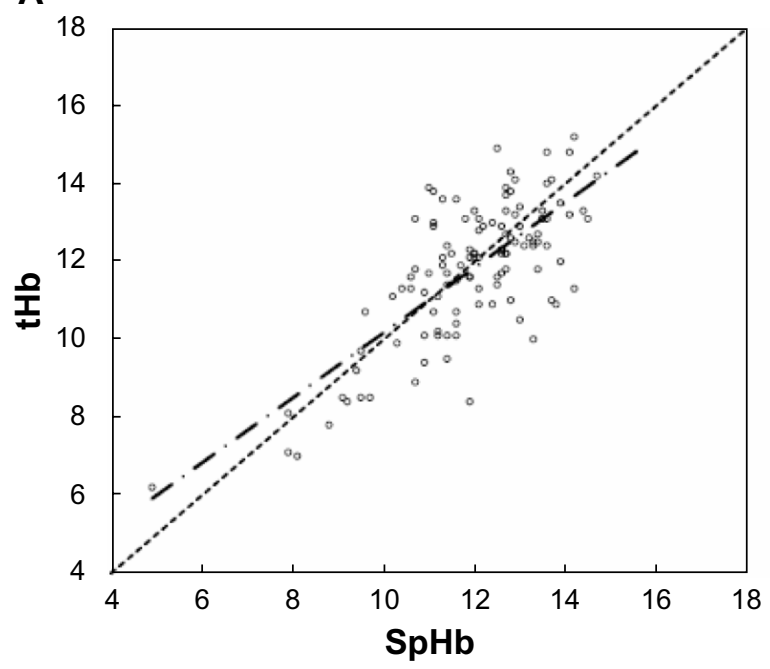

B

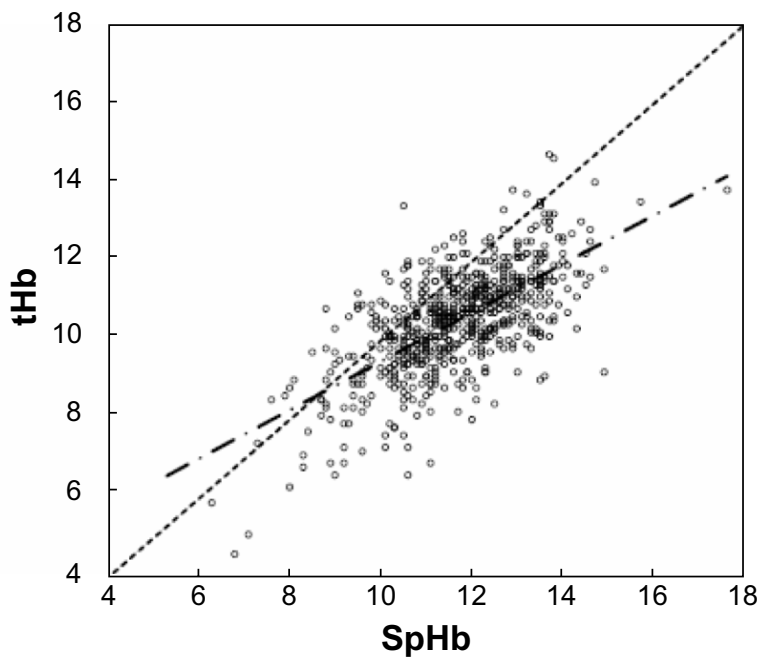

Figure 3 Scattergram of laboratory thb and $\mathrm{SpHb}$ measurements.

Notes: The broken lines are drawn at $\mathrm{SpHb}=\mathrm{tHb}$, and chained lines demonstrate the regression lines in both categories. (A) Nonpregnant and first trimester of pregnancy. The plots were located around the broken line. (B) Second and third trimester of pregnancy and postpartum period. The majority of the plots were located under the broken line, indicating the tendency of $\mathrm{SpHb}>\mathrm{tHb}$.

Abbreviations: $\mathrm{SpH}$, percutaneous hemoglobin; $\mathrm{tHb}$, total hemoglobin.

small. In this study, all the postpartum measurements were made before the seventh day of delivery. For the purpose of a longitudinal analysis of bias changes over the postpartum course, we do not have enough Pronto-7 data. We speculate that the bias may return to the nonpregnant level following the hemodynamic change occurring in the postpartum period. As of this writing, we have only eight measurements after 1 month postpartum ( $50 \pm 18$ postpartum days). The mean bias of those cases was $0.2 \pm 0.9 \mathrm{~g} / \mathrm{dL}$ (data not included in this report).

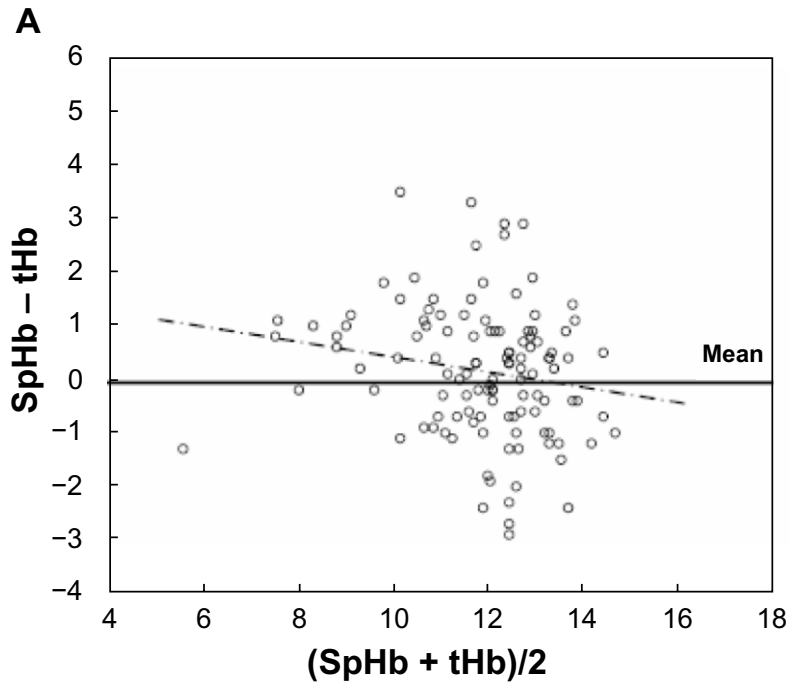

Hadar et al reported the accuracy of noninvasive hemoglobin measurement during pregnancy with a device (NBM-200; OrSense, Petah-Tikva, Israel) that was different from the device used in our study. ${ }^{17}$ In their report, the mean bias of SpHb levels with the NBM-200 was $0.1 \mathrm{~g} / \mathrm{dL}$, and the SD was $0.86 \mathrm{~g} / \mathrm{dL}$ in 63 pregnant women. They concluded that the NBM-200 could accurately and noninvasively assess hemoglobin levels during pregnancy. However, we think that their study has some limitations: 1) the number of the subjects was relatively small; 2) the

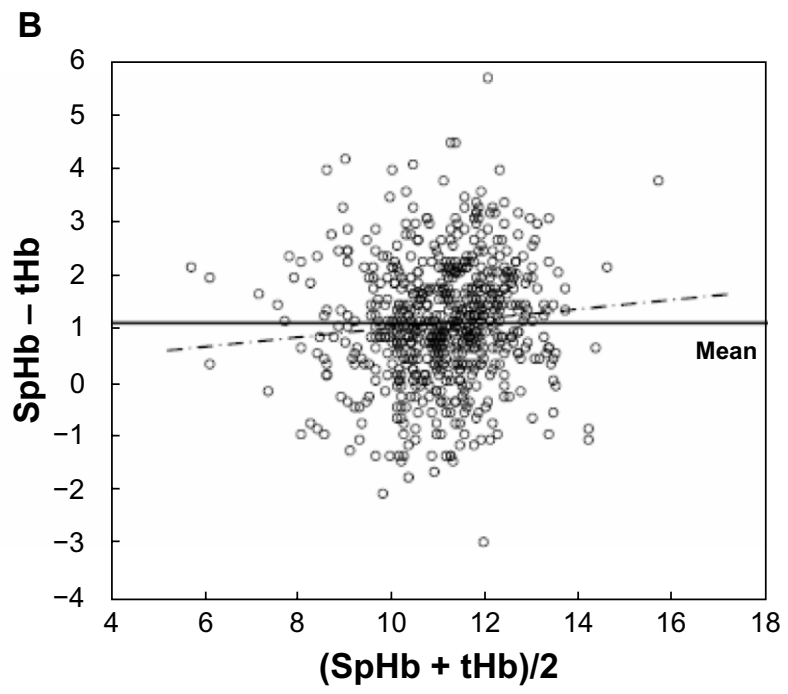

Figure 4 Bland-Altman comparison of $\mathrm{SpHb}$ and $\mathrm{tHb}$ of the two categories.

Notes: Horizontal lines are drawn at the mean bias, and horizontal broken lines are drawn at the limits of agreement. The chained lines demonstrate the regression lines in both categories. (A) Nonpregnant and first trimester of pregnancy. (B) Second and third trimester of pregnancy and postpartum period.

Abbreviations: $\mathrm{SpH} b$, percutaneous hemoglobin; $\mathrm{tHb}$, total hemoglobin. 
researchers grouped all data throughout pregnancy together and did not take into account the period of gestation; 3 ) the range of laboratory hemoglobin measurements was relatively narrow (6.9-13.9), and very severe anemic cases were not included; and 4) postpartum data were not included. With the possibility of hemorrhage at delivery, it is very important that the device be useful for the assessment of postpartum anemia; therefore, postpartum data must be included for the consideration of obstetrical utility. Further studies are necessary if we are to really use the NBM-200 analyzer instead of laboratory examination.

Compared with the NBM-200, the Pronto-7 has the advantage of portability. The size is smaller, and the weight is lighter, and it works on batteries. Importantly, at this point, the NBM-200 has not been approved for clinical use by the Ministry of Health, Labor and Welfare of Japan. More detailed studies are necessary for the assessment of the accuracy of the Pronto-7. We think that the accuracy of the $\mathrm{SpHb}$ measured by the Masimo Pronto-7 is almost high enough to merit its clinical use outside of pregnancy or in the first trimester of pregnancy. However, in the second and third trimesters of pregnancy and in early postpartum period, we think the $\mathrm{SpHb}$ measured with this device is not accurate enough.

In summary, the Masimo rainbow Pronto-7 may have clinical utility as a device to provide real-time and noninvasive assessments of hemoglobin level. However, in our current study, we observed higher hemoglobin values measured with this device than those measured in the conventional laboratory. We consider that currently, the device has certain limitations in obstetrical utility and requires modifications for use in the perinatal period.

\section{Disclosure}

The authors report no conflicts of interest in this work.

\section{References}

1. Berg CJ, Callaghan WM, Syverson C, Henderson Z. Pregnancy-related mortality in the United States, 1998 to 2005. Obstet Gynecol. 2010;116(6):1302-1309.

Medical Devices: Evidence and Research

\section{Publish your work in this journal}

Medical Devices: Evidence and Research is an international, peerreviewed, open access journal that focuses on the evidence, technology, research, and expert opinion supporting the use and application of medical devices in the diagnosis, treatment and management of clinical conditions and physiological processes. The identification of novel
2. Cantwell R, Clutton-Brock T, Cooper G, et al. Saving Mothers' Lives: Reviewing maternal deaths to make motherhood safer:2006-2008. The Eighth Report of the Confidential Enquiries into Maternal Deaths in the United Kingdom. BJOG. 2011;118 Suppl 1:S1-S203.

3. Knight M, Callaghan WM, Berg C, et al. Trends in postpartum hemorrhage in high resource countries: a review and recommendations from the International Postpartum Hemorrhage Collaborative Group. BMC Pregnancy Childbirth. 2009;9:55.

4. Nagaya K, Fetters MD, Ishikawa M, et al. Causes of maternal mortality in Japan. JAMA. 2000;283(20):2661-2667.

5. Duthie SJ. Postpartum hemorrhage in Asian countries: available data and interpretation. In: Lynch CB, Keith LG, Lalonde AB, Karoshi MA. A Textbook of Postpartum Hemorrhage. Kirmahoe: Sapiens Publishing; 2006:453-461.

6. Japan Association of Obstetricians and Gynecologists. [Maternal deaths in pregnancy]. Available from: http://www.jaog.or.jp/all/ document/67_130710.pdf. Accessed December 6, 2013. Japanese.

7. Prasertcharoensuk W, Swadpanich U, Lumbiganon P. Accuracy of the blood loss estimation in the third stage of labor. Int J Gynaecol Obstet. 2000;71(1):69-70.

8. Raikhel M. Accuracy of noninvasive and invasive point-of-care total blood hemoglobin measurement in an outpatient setting. Postgrad Med. 2012;124(4):250-255.

9. Isosu T, Obara S, Hosono A, et al. Validation of continuous and noninvasive hemoglobin monitoring by pulse CO-oximetry in Japanese surgical patients. J Clin Monit Comput. 2013;27(1):55-60.

10. Frasca D, Dahyot-Fizelier C, Catherine K, Levrat Q, Debaene B, Mimoz O. Accuracy of a continuous non-invasive hemoglobin monitor in the ICU. Critical Care. 2011;15(Suppl 1):S151-S152.

11. Masimo [homepage on the Internet]. Available from: http://www. masimo.com/. Accessed December 6, 2013.

12. Ghys T, Malfait R, VAN den Bossche J. Performance evaluation of the Sysmex XS-1000i automated haematology analyser. Int J Lab Hematol. 2009;31(5):560-566.

13. Bland JM, Altman DG. Statistical methods for assessing agreement between two methods of clinical measurement. Lancet. 1986;1(8476): 307-310.

14. Butwick A, Hilton G, Carvalho B. Non-invasive haemoglobin measurement in patients undergoing elective Caesarean section. $\mathrm{Br} J$ Anaesth. 2012;108(2):271-277.

15. Macknet MR, Allard M, Applegate RL, Rook J. The accuracy of noninvasive and continuous total hemoglobin measurement by pulse COOximetry in human subjects undergoing hemodilution. Anesth Analg. 2010;111(6):1424-1426.

16. Miller RD, Ward TA, Shiboski SC, Cohen NH. A comparison of three methods of hemoglobin monitoring in patients undergoing spine surgery. Anesth Analg. 2011;112(4):858-863.

17. Hadar E, Raban O, Bouganim T, Tenenbaum-Gavish K, Hod M. Precision and accuracy of noninvasive hemoglobin measurements during pregnancy. J Matern Fetal Neonatal Med. 2012;25(12):2503-2506.

\section{Dovepress}

devices and optimal use of existing devices which will lead to improved clinical outcomes and more effective patient management and safety is a key feature. The manuscript management system is completely online and includes a quick and fair peer-review system. Visit http://www. dovepress.com/testimonials.php to read real quotes from authors. 